Z. klin. Chem. u. klin. Biochem.

7. Jg., S. 183-184, März 1969

\title{
Messung von sehr geringen $o$-Toluidin-Konzentrationen am Arbeitsplatz für die Blutzuckerbestimmung
}

\author{
Von A. Härtel, R. Helger und H. Lang \\ Aus der Biochemischen Abteilung der E. Merck AG., Darmstadt
}

(Eingegangen am 11. Februar 1969)

Bei routinemäßiger Durchführung der Blutzuckerbestimmung mit dem o-Toluidin-Reagenz besteht kein realer Anhalt für eine Gefährdung der Laboranten durch o-Toluidin-Dämpfe. Selbst unter extrem ungünstigen Bedingungen liegen die o-Toluidin-Dampfdichten noch weit unterhalb der festgelegten MAK-Werte.

\section{Determination of very small concentrations of o-toluidine in the laboratory for blood sugar determination}

There is no evidence that the routine determination of blood sugar with o-toluidine reagent constitutes a hazard to the personel concerned. Even under the most unfavourable conditions, the concentration of 0 -toluidine vapour is far below the maximum permitted level.

Bei der Blutzuckerbestimmung mit dem o-ToluidinEisessigreagenz soll nach BürgI und MTTTELHOLZER (1) die Gefahr einer Vergiftung durch o-Toluidin bestehen. Von den angegebenen Vergiftungs-Möglichkeiten ist das Einatmen der Dämpfe durch das Laborpersonal am schwierigsten $\mathrm{zu}$ vermeiden. Als tolerierte Luftkontamination geben BürGI und MitTELHOLZER den für o-Toluidin festgesetzten MAK-Wert ${ }^{1}$ ) von $5 \mathrm{ppm}$ $(=22 \mu \mathrm{g} / L)(2,3)$ an. Die Autoren lassen aber die Frage offen, wie hoch die 0 -Toluidin-Konzentrationen in der Laborluft bei routinemäßiger Durchführung der Methode und unter extremen Bedingungen tatsächlich sind. $\mathrm{Da}$ über sauren Lösungsmitteln die Dampfdichten von gelösten Basen wegen der Salzbildung äußerst gering sind, erscheint es fraglich, ob überhaupt toxische Konzentrationen erreicht werden können. Im Hinblick auf die weite Verbreitung der Methode und die zur Zeit stattfindende Diskussion. sollte die Frage einer angeblichen Vergiftungsgefahr jedoch genau untersucht werden.

\section{Versuchsbeschreibung \\ Messung der o-Toluidin-Dampfdicbte in der Laborluft}

3 Waschflaschen werden mit je $50 \mathrm{ml}$ iso-Propanol gefüllt und hintereinander geschaltet. (PVC-Scbläuche sind zur Verbindung ungeeignet, $\mathrm{da}$ Weichmacher herausgelöst werden; gut geeignet sind Silikonschläuche.) Dann saugt man Luft $(20-80 \mathrm{l})$ mit einer Geschwindigkeit von $1-3 \mathrm{~m} / /$ Sek hindurch und mißt anschließend das Volumen. Um Kondensattröpfchen, die sich eventuell im Ansaugrohr abgesetzt haben, mitzuerfassen, wird durch das Ansaugrohr mehrfach etwas iso-Propanol gespült. Man mißt das UV-Spektrum der Waschlösungen gegen reines iso-Propanol und bexechnet aus der Höhe des o-Toluidin-Extinktionsmaximums $(284 \mathrm{~nm})$ die o-Toluidin-Konzentrationen. Da die spezifische Extinktion vom Eisessiggehalt der Lösung abhängt, verwendet man zur Eichung eine iso-Propanol-Lösung, die außer der bekannten Menge o-Toluidin noch eine der unbekannten Probe entsprechende

1) Der MAK-Wert ist diejenige maximale Konzentration eines Gases, Dampfes oder Staubes in der Luft eines Arbeitsraumes, in Atemhöhe gemessen, die bei täglich $8 \mathrm{stdg}$. Inhalation ohne irgendwelche gesundheitliche Nachteile auch bei jahrelanger Exposition vertragen wird.
Eisessigmenge enthält. Diese Eisessigmenge wird durch Titration ermittelt.

\section{Messung der o-Toluidin-Sättigungsdampfdichte}

3 Waschflaschen werden mit je $50 \mathrm{ml}$ o-Toluidin-Reagenz bzw. mit der freien Base gefüllt. Außerdem gibt man einen mit dem Reagenz bzw. mit der Base gewaschenen Glaswollebausch noch feucht in die Waschflaschen. Die dritte Waschflasche enthält ein kleines Thermometer, das von außen abgelesen werden kann. Diese 3 Waschflaschen werden vor die mit iso-Propanol gefüllten Waschflaschen geschaltet. In Analogie zum vorstehend beschriebenen Versuch mißt man jetzt die o-Toluidin-Sättigungsdampfdichte über dem Blutzucker-Reagenz bzw. über der freien Base.

\section{Kontrolle der Meßanordnung}

Zur Kontrolle der Sättigung werden Versuche mit unterschiedlich vielen Waschflaschen mit o-Toluidin-Reagenz durchgeführt. $\mathrm{Da}$ die Ergebnisse bei Verwendung von 2 oder mehr Waschflaschen unabhängig von der $\mathrm{Zahl}$ der Flaschen sind, ist die hindurchströmende Luft mit $o$-Toluidin gesättigt.

Das vollständige Auswaschen durch die iso-Propanol-Waschflaschen wird durch Messungen der 0 -Toluidin-Konzentrationen in den einzelnen Waschflaschen kontrolliert. $\mathrm{Da}$ die erste isoPropanol-Flasche mehr als $98 \%$ des übergangenen o-Toluidins, die $z$ weite weniger als $2 \%$ und die dritte überbaupt kein 0 -Toluidin enthält, wird das o-Toluịin vollständig durch die angegebene Anordnung erfaßt.

Zur weiteren Kontrolle wird der o-Toluidin-Sättigungsdampfdruck über der freien Base, der im Gegensatz zu dem über dem Blutzucker-Reagenz aus der Literatur bekannt ist, bei verschiedenen Temperaturen bestimmt. Es ergibt sich eine gute Übereinstimmung mit den Daten aus der Literatur, so daß die gemessenen Werte als zuverlässig anzusehen sind.

\section{Modellarbeitsplatz}

Im Hinblick auf die angebliche Vergiftungsgefahr durch o-Toluidin-Dämpfe sind Messungen in der Atemluft bei routinemäßiger Blutzuckerbestimmung erforderlich. Als Modellarbeitsplätze wurden 2 kleine Labotatorien mit geschlossenen Fenstern und Türen gewählt. Eines dieser Laboratorien enthielt keine Lüftung, das andere eine Raumlüftung, aber keinen Abzug. Da die stärksten o-Toluidin-Dampfkonzentrationen über den offenen, mit Reagenz gefüllten Reagenzgläsern im siedenden Wasserbad zu crwarten sind, wurdè das Ende des Ansaugrohres $8 \mathrm{~cm}$ über den 20 Gläscrn angebracht. In einem weiteren Versuch wurde die Luft $80 \mathrm{~cm}$ von den Gläsern entfernt in Kopfhöhe entnommen. $\mathrm{Da}$ der Gehalt an 0 -Toluidiniumsalz im Reagenz in der Siedehitze langsam abnimmt, wurden alle mit je $2 \mathrm{~m} /$ Reagenz gefüllten Reagenz- 
gläser nach jeweils $15 \mathrm{Min}$. durch frische ersetzt. Den Beginn der Luftentnahine setzten wir auf $3 \mathrm{Stdn}$. nach Versuchsanfang fest. In cinem weiteren Versuch, der das Verdampfen von o-ToluidinRengenz beim $\Lambda$ btupfen der Küvetten am Photometer und beim Herumliegenlassen von reagenzbeschmutzten Glasgeräten simulieren sollte, wurde cin Celluloselappen $(20 \times 20 \mathrm{~cm})$ auf eincr Glasplatte ausgebreitet und mit Reagenz befeuchtet. Nach je 15 Min. wurde neues Rengenz auf den Lappen gegeben. Dic 0Toluidin-Kon\%entration wurde $8 \mathrm{~cm}$ und außerdem $80 \mathrm{~cm}$ vom Lappen entfernt 3 Stdn. nach Beginn des Versuches bestimmt. Zu dicsem Zeitpunkt war ein längerer Aufenthalt im Raum wegen des außcrordentlich starken Eisessiggeruchs nicht möglich. Obwohl wir scit viclen Jahren dic Blutzuckerbestimmung mit der 0 Toluidin-Methode durchführen, sind derartige Eiscssigkonzentrationen in der Laborluft nie vorgekommen. Dieser Modellarbeitsplatz würde also im Hinblick auf dic angebliche Vergiftungsgefahr extreme Anforderungen an das Personal stellen.

\section{Ergebnisse}

Eine große Zahl von Dampfdichtebestimmungen am Arbeitsplatz zeigt, daß die von BürgI und MrtrezHolzer angegebene Toleranzgrenze in der Praxis bei weitem nicht erreicht wird. Selbst die Sättigungsdampfdichte bei Zimmertemperatur liegt noch unter dem angegebenen MAK-Wert. Die Mittelwerte der verschicdenen Meßreihen sind in Tabelle 1 angegeben.

Tab. 1

o-Toluidin-Dampfdichte in der Laborluft am Arbeitsplatz für die Blutzuckerbestimmung $\left(2^{\circ}\right)$

\begin{tabular}{|c|c|c|c|}
\hline \multirow[t]{2}{*}{ Ort der Luftentnahme } & \multicolumn{3}{|c|}{ o-Toluidin-Dampfdichte } \\
\hline & $\begin{array}{c}\text { Labor init } \\
\text { Raumlüftung } \\
\text { /g/l }\end{array}$ & $\begin{array}{c}\text { Labor ohne } \\
\text { Raumlüftung } \\
\mu \mathrm{g} / l\end{array}$ & $\begin{array}{c}\text { freien } \\
\text { Base } \\
\text { } \mu \mathrm{g} / l\end{array}$ \\
\hline
\end{tabular}

$8-10 \mathrm{~cm}$ über der

Offnuting der Reagenz-

gläser in siedendell

Wasserbad

in Kopfhühe,

$60-80 \mathrm{~cm}$ von den

Reagenzgläsern im

siedenden Wasserbad
entfernt

$10 \mathrm{~cm}$ über cinem

mit Reagenz

getränkten Lappen $\quad 0,5$.

$80 \mathrm{~cm}$ über cincm

mit Reagenz

getrünkten Lappen $\quad 0,5$

geshittigter Raunı

Tolerimzerenze nacl

BUROI (MAK-Wert)

1,4

5,6

0,1

2,0

14

22

\section{Diskussion}

Die o-'Toluidin-Methode zur Blutzuckerbestimmung hat in den letzten Jahren wegen ihrer guten Spezifität, der greringen Störanfälligkeit und der einfachen Durch- führung die früher gebräuchlichen nichtenzymatischen Methoden weitgehend abgelöst. Die Brauchbarkeit ist durch eine große Zahl von Veröffentlichungen belegt. Wie die hier durchgeführten Messungen zeigen, werden toxische 0 -Toluidin-Konzentrationen in der Atemluft auch unter extrem ungünstigen. Umständen bei weitem nicht erreicht, da die 0-Toluidin-Dampfdichte über dem Reagenz im Gegensatz zu derjenigen über der freien Base sehr gering ist. Nun wird aber wegen der schwierigen Reinigung der freien Base fast ausschließlich das haltbare Reagenz (in Eisessiglösung) gebrauchsfertig vom Hersteller bezogen. Deshalb ist uns trotz der weiten Verbreitung der Methode bisher kein einziger Vergiftungsfall aus der Literatur oder durch Hinweise aus einem der vielen Laboratorien, mit denen wir in Kontakt stehen, bekannt geworden. Der von BürGI und Mittelholzer gegebene Hinweis kann deshalb nur für die wenigen Laboratorien gelten, in denen das Reagenz selbst angesetzt wird.

Wenn über die Luftverunreinigung am Arbeitsplatz bei der Durchführung der o-Toluidin-Methode gesprochen wird, darf man den Essigsäuredampf nicht vergessen. Der MAK-Wert für Eisessig beträgt $25 \mu \mathrm{g} / /(2,3)$ und liegt damit erheblich unter der Sättigungsdampfdichte von $100000 \mu \mathrm{g} / / 25^{\circ}$ (4). Da die Erkennungssch chwelle für Essigsäure jedoch bereits bei $1,6 \mu \mathrm{g} / \mathrm{l}$ (5) liegt, kommt es in der Praxis wegen der bei höheren Konzentrationen starken Geruchsbelästigung nicht zu Vergiftungen. Man kann aber auch diese Geruchsbelästigung durch Verwendung des eisessigfreien o-ToluidinReagenzes ${ }^{2}$ ) vermeiden (6).

Im Zusammenhang mit der Möglichkeit einer Vergiftung durch das o-Toluidin-Reagenz muß ferner noch auf die vor längerer Zeit aufgeworfene Frage nach der Carcinogenität eingegangen werden. Nach den Untersuchungen von SCHMÄHL $(7,8)$ besteht für eine Gefährdung der betreffenden Laboranten hinsichtlich einer möglichen carcinogenen Wirkung durch den Umgang mit o-Toluidin kein realer Anhalt. Der Gebrauch des o-Toluidin-Blutzuckerreagenzes erscheint beim Einhalten der entsprechenden Arbeitsvorschriften unbedenklich.

Wir danken Fräulein R. Finkenauer, Frau A. Himsel und Fräulein H. NaEve für die Durchführung der Versuche.

2) Merckotest Blutzucker (o-Toluidin ohne Essigsäure) Artikel Nr. 3350.

\section{Literatur}

1. Blimgil, WW. und M. L. Mitteliolzer, Praxis 57, 3 (1968). 2. Moisiutuin, S., Klinik und Thernpic der Vergiftungen, 4. Aull. S. 12. Gcorg-T'hicme-Verlag, Stuttgart, (1964). - 3. Bekantunachung des Bundesministeriums für Arbeit vom 15.10. 1968 - II1 I) 4 - 3745-81 - 4200/68. - 4. LANDOLT-BöRNSTEIN, Zahlentverte und liunktionen aus Physik, Chemic, Astronomic, Gcophysile und 'I'echurik, II. Band, 2. 'Teil, Bandteil a', S. 202. Springer-Verlag, Berlin-Göttingen-Heidelberg (1960). - 5.
Morimura, S., Tohoku J. exp. Med. 22, 417 (1933). -6. Härtel, A., R. Helger und H. LANG, diese Z. 7, 14 (1969). - 7.DruCKREY, H., D. Schmähl und A. Retrer, Arzneimittelforsch. Aulendorf 4, 365 (1954). - 8. Prof. Dr. D. SchmäHL, Direktor des Instituts für exp. Geschwulsterzeugung und Geschwralstbehandlung im Deutschen Krcbsforschungszentrum, Heidelberg. Gutachtliche Außerung vom 16.12. 1968.
Dr. A. Härtel, Biochemische Abteilung E. Merck AG

61 Darmstadt, Postfach 4119 
Fresenius' Zeitschrift für
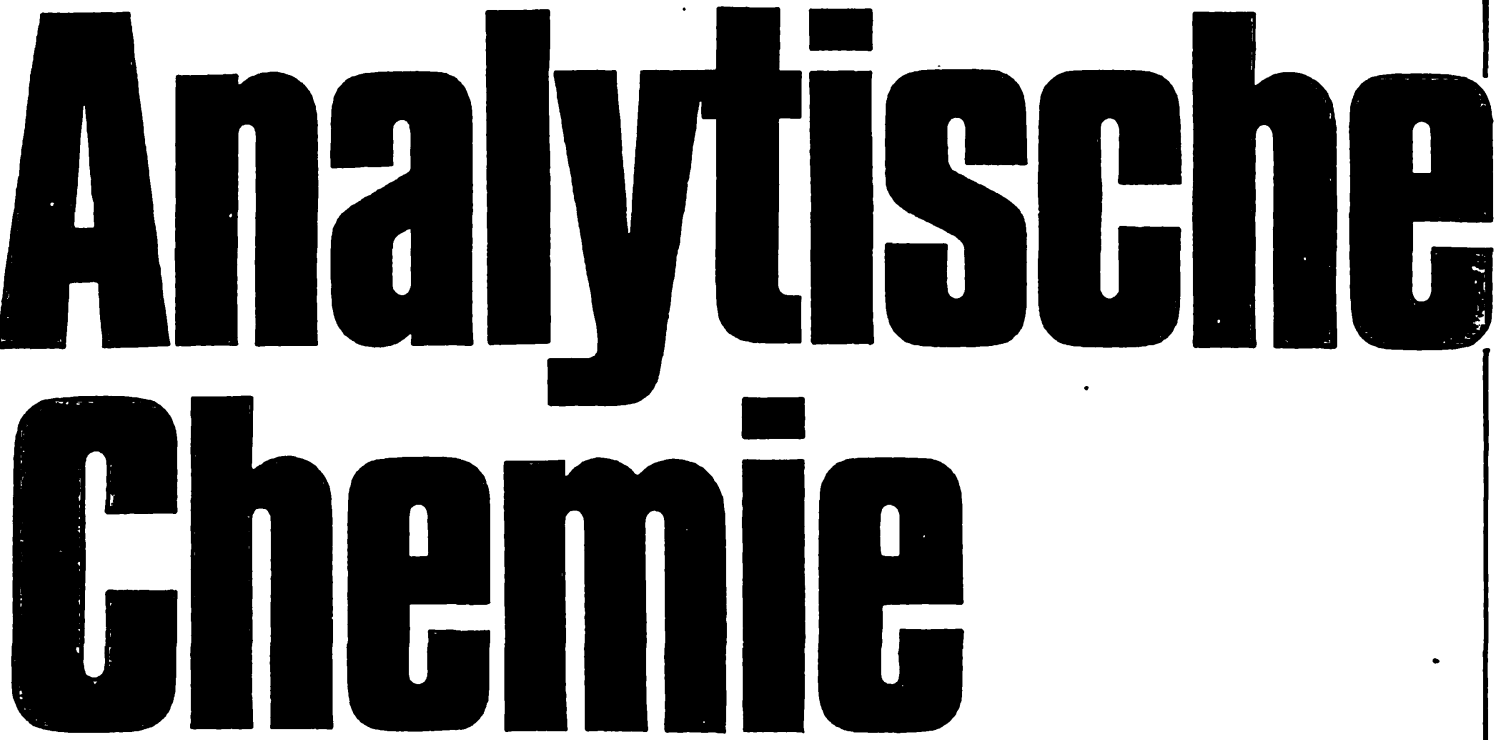

\title{
Labor- und Betriebsverfahren
}

\section{Herausgegeben von W. Fresenius}

\author{
Unter Mitwirkung der Fachgruppe Analytische Chemie \\ der Gesellschaft Deutscher Chemiker \\ vertreten durch $H$. Bode, $H$. Kienitz, W. Koch, \\ H. Luther, H. Specker
}

Ab 1969

14 täglich im ${ }^{2}$ neuen

Format
Die "Zeitschrift für Analytische Chemie" spiegelt den Weg der Analytik von den klassischen Verfahren zu den modernen physikalisch-chemischen Analysenmethoden wider. Meilensteine der Chemiegeschichte sind die Publikationen von $G$. Kirchhoff und $R$. Bunsen, von R. Fresenius, Fr. Mohr und G. F. Schönbein, die bereits im 1862 erschienenen ersten Band mit Arbeiten vertreten sind. Die wachsende Bedeutung der Analytik für die Industrie, das chemische Untersuchungslaboratorium, die biochemische Forschung, für klinische Untersuchungen und Forschungsaufgaben der organischen Chemie, läßt jeder neue Band erkennen.

$\mathrm{Zu}$ den Autoren und Lesern zählen Chemiker aus aller Welt; die Zeitschrift wird in etwa 60 Ländern verbreitet; in der UdSSR und in China (beide Länder haben sich der Berner Konvention nicht angeschlossen) wird sie nachgedruckt. Ungefähr ein Drittel der Originalarbeiten erscheint derzeit in englischer Sprache.

Die Aktualität der Zeitschrift zeigt sich nicht nur in der raschen Veröffentlichung von Kurzmitteilungen, sondern auch in der ausführlichen Berichterstattung über Tagungen internationaler Gesellschaften. Besonders interessierende Themen werden von Zeit zu Zeit in zusammenfassenden Berichten behandelt.
Im Referate-Teil werden analytisch wichtige Arbeiten aus rund 300 Zeitschriften laufend ausgewertet, und zwar in einer solchen Form, daß es dem Analytiker im allgemeinen möglich ist, direkt aufgrund des Referates die beschriebenen Methoden zu erproben und anzuwenden. Der weitere Ausbau des ReferateTeils der Zeitschrift stellt einen Beitrag zum Gesamtgebiet der chemischen Dokumentation dar.

Ausführliche Register erschließen nicht nur die Originalarbeiten und Kurzen Mitteilungen, sondern ebenso die Referate. Die Zëitschrift ist daher seit vielen Jahrzehnten ein geschätztes Nachschlagewerk. Generalregister erscheinen in Perioden von etwa zwei Jahren.

Auch künftig wird über die Tätigkeit der Fachgruppe Analytische Chemie der Gesellschaft Deutscher Chemiker, die an der Herausgabe der Zeitschrift mitwirkt, berichtet werden. Die Rubrik ${ }_{n}$ Neue Geräte und Chemikalien", die sich seit einigen Jahren besonderer Beliebtheit erfreut, wird weitergeführt. 1969 hat die Zeitschrift mit einem neuen Format gleichzeitig ein modernes Layout erhalten. Sie ist damit nicht nur leichter lesbar, sondern auch übersichtlicher geworden. - Probehefte können beim Verlag kostenlos angefordert werden.

1969 erscheinen voraussichtlich 5Bände (Bde. 244-248) Preis eines Bandes DM 96,-; US $\$ 24.00$ zuzüglich Porto.

\section{Springer-Verlag Berlin Heidelberg New York}

\section{J. F. Bergmann München}




\title{
BULLETIN
}

\section{DE LA SOCIÉTÉ DE CHIMIE BIOLOGIQUE}

\author{
(„Berichte der Gesellschaft für biologische Chemie“") \\ Unter Mitwirkung des \\ „CENTRE NATIONAL DE LA RECHERCHE SCIENTIFIQUE“ \\ (National-Centrum für wissenschaftliche Forschung) veröffentlicht
}

R. PERLÈS

Hilfs-Generalsekretär

\section{J. E. COURTOIS}

Generalsekretär
Y. RAOUL

Hauptredakteur

Seleretariat und Redaletion

4, avenue de l'Observatoire, Paris $\left(6^{\mathrm{e}}\right)$

Herausgeber:

Masson et $\mathrm{C}_{\mathrm{IE}}, 120$, Boulevard Saint-Germain, Paris (6 $\left.6^{\mathrm{C}}\right)$

Der „Bulletin de la Socí́té de Chimie Biologique“ veröffentlicht jährlich $11 \mathrm{Hefte;} \mathrm{diese} \mathrm{enthalten} \mathrm{die}$ Arbeiten der französischen Biochemiker, welche der „SocrétÉ DE CHImre Brologrquz“ (Gesellschaft für biologische Chemie) angehören.

Abonnementspreis 1969:

Frankreich und „Franc-Zone“ . . 150 francs

Belgien . . . . . . . . 1684 belges

Andere Länder . . . . . . 165 francs

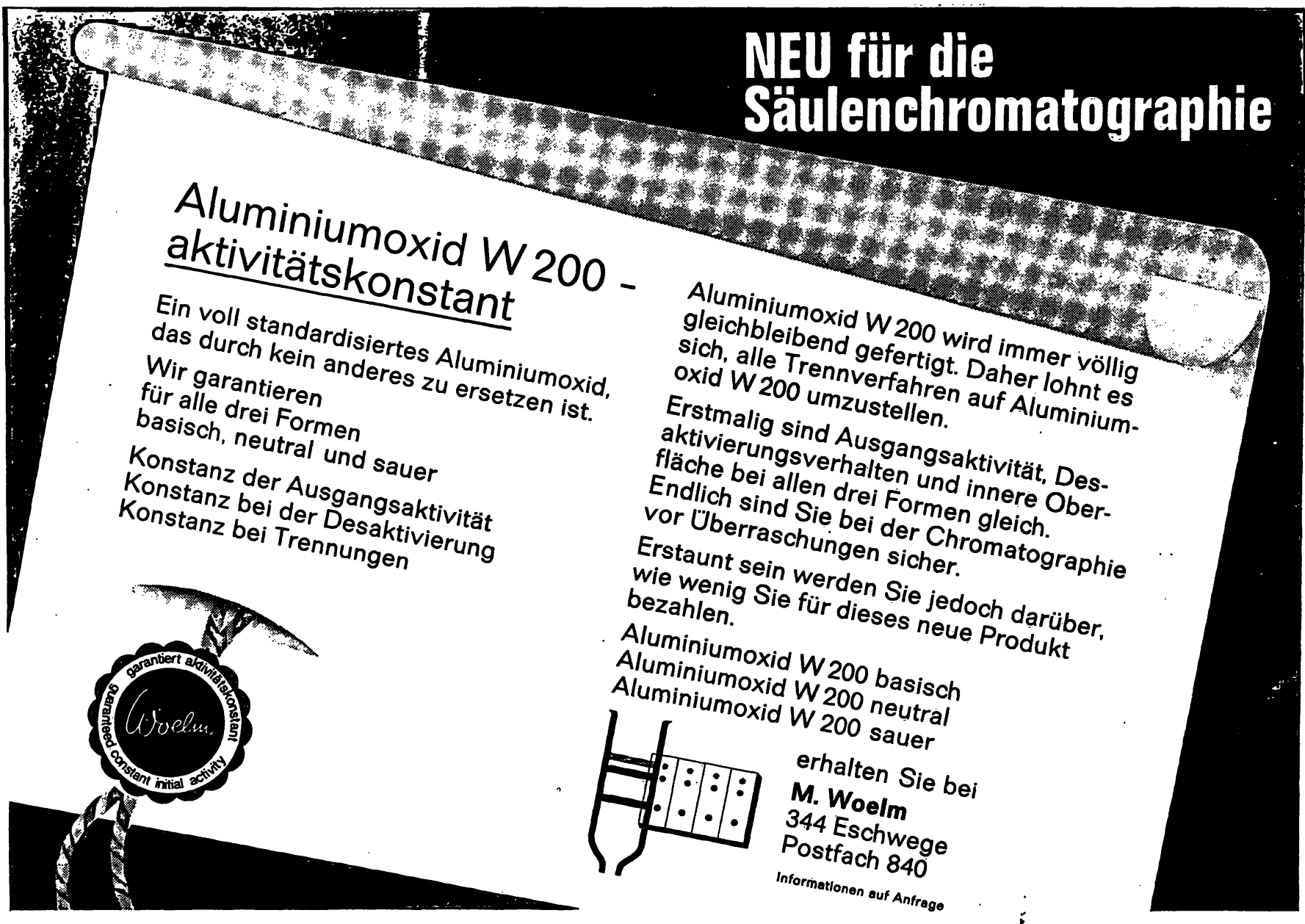


Solen erschien:

Heinzgeorg Vogelsang *

\section{Die spinale Ossovenographie}

Eine diagnostische Methode

zur Erkennung patbologischer Prozesse der Wirbelsäule und des Spinalkanales

Mit einem Geleitwort von Prof. Dr. H. W. PrA

Mit 122 Abbildungen und 11 Tabellen.

Groß-Oktav. 123 Seiten. 1969.

Plastik flexibel DM 42,-

Basierend auf einer verbesserten Untersucbungstechnik und systematischen Anvendung bei Erkrankungen der Wirbelsäule (Bandscheibenvorfälle, Spondylolisthesis, traumatischen Veränderungen, primären und ;ekundären Tumoren, Spondylosis deformans u. a.) sowie des Spinaltanales (raumfordernde Prozesse, Gefäßmißbildungen, entzündiiche Veränderungen) werden die Erfahrungen niedergelegt, die Jer Verfasser mit der transossalen Phlebographie sammeln konnte. Grundziige der Anatomie und Physiologie der Venensysteme, Methodik, Normalbefunde, Indikation und Kontraindikation, Komplikationen und Grenzen der Methode verden abgebandelt, batbologiscbe Befunde bei den einzelnen Krankheitsgruppen dargestellt und diskutiert.

133 Abbildungen und Tabellen veranschaulichen die Ausführungen. Vergleiche mit den üblichen Röntgenaufnahmen, myelographischen Untersuchungen und bioptischen Befunden zeigen eindrucksvoll die Leistungsfähigkeit und -breite dieser angiographischen Methode.

* Privatdozent Dr. med., Leiler der Neuroradiologiscben Abteilung an der Neurocbirurgiscben Universilattsklinik Gießen

Walter de Gruyter \& Co - Berlin 30
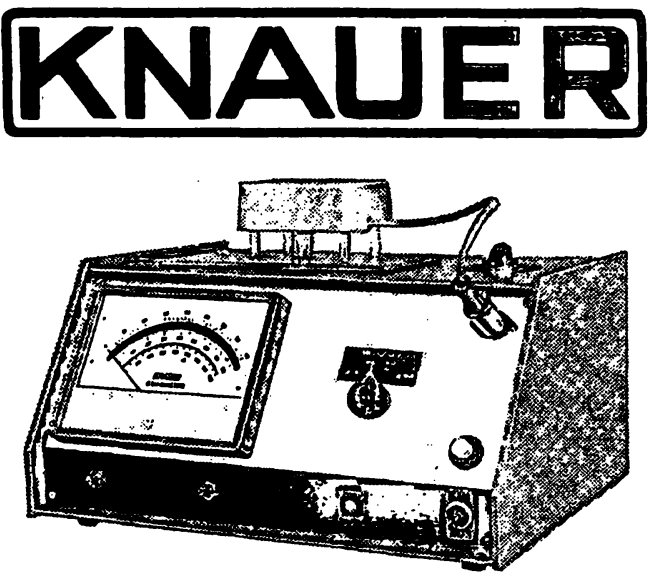

ELEKTRONISCHES HALBMIKRO-OSMOMETER

zur direkten Bestimmung der Os mol a lität aller Körperflüssigkeiten wie Blut, Serum, Urin, Liquor durch Gefrierpunktmessung.

- Probevolumen nur $0,15 \mathrm{ml}$ oder $0,05 \mathrm{ml}$

- Messdauer ca. 2 Minuten

- Genauigkeit und Reproduzierbarkeit 1-2 Millios$\mathrm{mol} / \mathrm{kg}$ bzw. $1 \%$

- Preis DM 3600,- + Mehrwertstever

Weitere Spezialität: Komplettes System zur Molekulargewichtsbestimmung zwischen 100 und 1000000 durch Kryoskopie, Dampfdruck-Osmometrie und Membran-Osmometrie.

Wissenschaftlicher Gerätebau

KG Dr. Ing. Herbert Knaver \& Co. GmbH

1 Berlin 37 (West) - Holstweg 18 - Telefon 848705

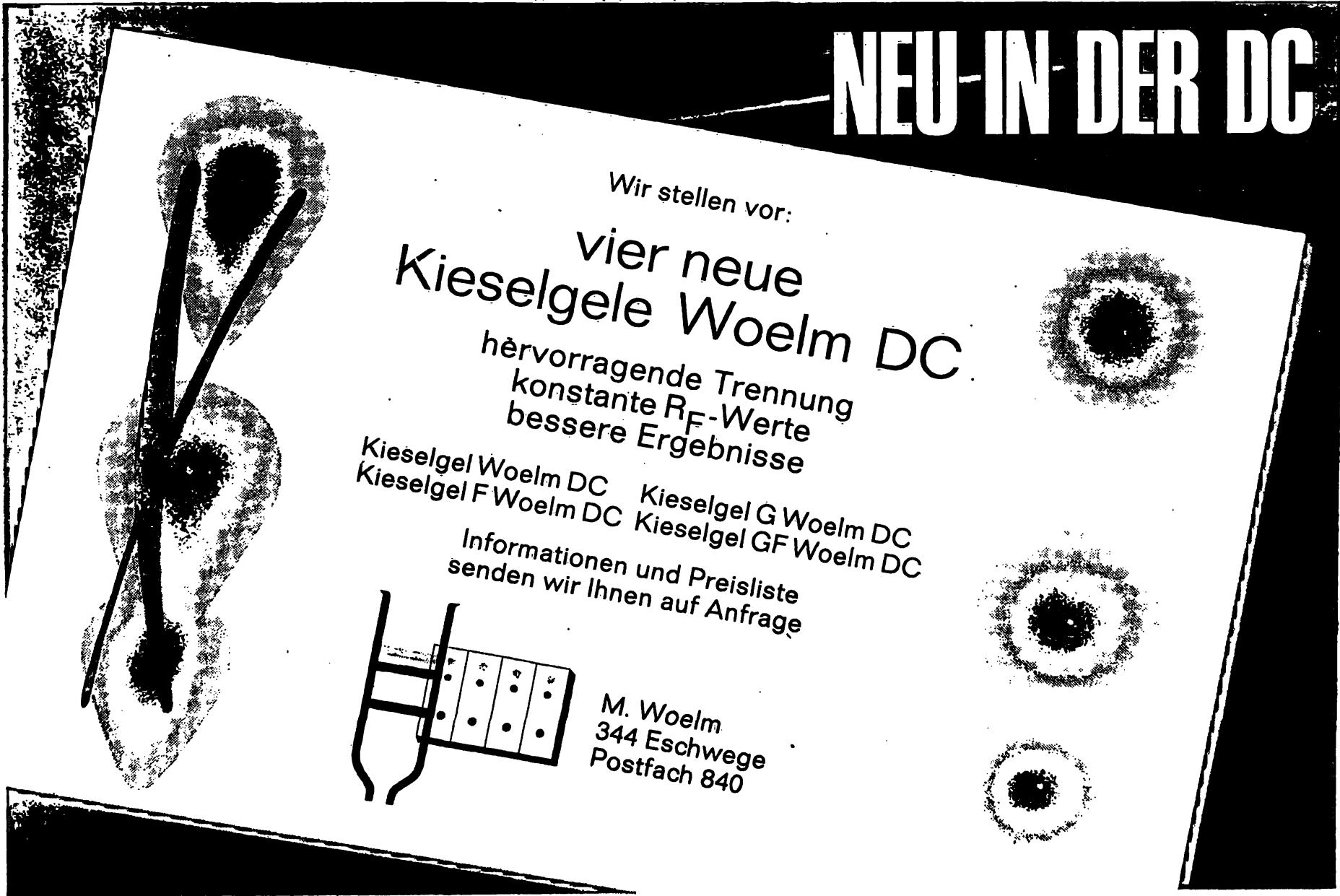


Groß-Oktav. XVI, 513 Seiten.

Mit 210 Abbildungen und

2 mehrfarbigen Tafeln.

1969. Ganzleinen DM 88,-
Herausgegeben von

Prof. Dr. med. Heinrich Bartelheimer Direktor der T. Medizinischen Universitäts-Klinik Hamburg

Prof. Dr. med. Hans-Joachim Maurer Institut und Klinik für Medizinische Strahlenkunde der Universität Düsseldorf

Prof. Dr. med. Hans W. Schreiber

Chefarzt der Chirurgischen Abteilung des Marienkrankenhauses, Hamburg

unter Mitwirkung von

Priv.-Doz. Dr. med. Kurt Müller-Wieland Oberarzt der I. Medizinischen Universitätsslinik Hamburg

Durch die Zusammenarbeit verschiedener Disziplinen und durch die Entwicklung neuer Methoden sind in der Gastrologie neue Erkenntnisse über Form und Funktion des gesunden und kranken Magens gewonnen worden. Die cbirurgischen Leistungen wurden durch die Einbeziehung internistischer, röntgenologischer, nuklearmedizinischer und vor allen Dingen biochemischer Verfabren ergänzt.

Gastroenterologen, Chirurgen, Röntgenologen, Hämatologen, Osteologen und Pädiater sowie Vertreter anderer Fächer haben aus diesen Gründen hier die Probleme der Magenoperation und der Magenoperierten dargestellt. Erst langjährige und vergleichende Erfahrungen gestatten eine kritische Bewertung, wie sie jetzt hier vorgelegt wird.

Mit Beiträgen von

H. Bartelhetmer - V. Becker - H. Berndt - K. Diwok - E. Farthmann - H. Frey-

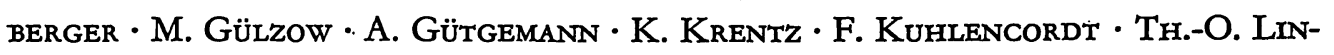
DENSCHMidt - A. LuChMANN - H.-J. MAURER - F. MEISSNER - K. MÜLLER-Wieland W. Pribilla - H.-J. Schreiber - F. Stelzner - K.-O. VorläNder - E. Wildhirt L. ZuCKSCHWERDT

Das Buch wendet sich an alle Disziplinen der Medizin.

Verbindliche und eindeutige Aussagen zur Diagnostik und zum therapeutischen Vorgehen finden der praktizierende Arzt, der die erste Indikation zu stellen hat, ebenso wie Gastroenterologen, Internisten, Cbirurgen und Röntgenologen. 